\title{
Femur Statistical Atlas Construction Based on Two-level 3D Non-rigid Registration
}

\author{
C. $\mathrm{Wu}^{*+}$, P. E. Murtha, $\mathrm{PhD}$, B. Jaramaz, $\mathrm{PhD}^{*}$ \\ chenyuwu@cmu.edu,PMurtha@icaos.org,branko@icaos.org \\ * Robotics Institute, School of Computer Science \\ Carnegie Mellon University, 5000 Forbes Avenue, Pittsburgh, PA 15213 \\ + Correspondence: Chenyu Wu, 201 Smith Hall, CMU, 5000 Forbes Avenue, Pittsburgh, PA 15213 \\ Note: This paper is based on the work published in 2nd International Conference on \\ Computer Vision Theory (VISAPP2007), Insticc Press, Barcelona, March 2007.
}

\begin{abstract}
The statistical atlas is a 3D medical image analysis tool towards more patient-oriented and more efficient diagnosis. The atlas includes information on geometry and their variation across populations. The comparison and information from other patients is very useful for the objective quantitative diagnosis. The statistical atlas can also be used to solve other challenging problems such as image segmentation. As a key to build statistical atlases, 3D registration remains an important yet unsolved problem in the medical image field due to the geometrical complexity of anatomical shapes and computational complexity caused by the enormous size of volume data.

Method: In this work we developed a two-level framework to efficiently solve 3D nonrigid registration and applied the method to the problem of building statistical atlases of femur. Compared with a general multi-resolution framework, we employed an interpolation to propagate the matching instead of repeating the registration scheme in each resolution. The registration procedure is divided into two levels: a low-resolution solution to the correspondences and mapping of surface models using Chui and Rangarajan's thin-plate spline (TPS) based algorithm [25], followed by an interpolation to achieve high-resolution matching. After that, principal component analysis (PCA) is used to build the statistical atlas. Experimental results show the shape variation learned from the atlas, and also demonstrate that out method significantly improves efficiency of registration without decreasing accuracy of atlases.
\end{abstract}

Keywords: Statistical atlas, Femur, Two-level, 3D Non-rigid Registration 


\section{Introduction}

Statistical atlases are used as references to interpret CT/MRI images [1], and represent the shape or appearance of human anatomical structures $[2,3]$. Some atlases are based on physical properties, such as elastic models [4, 5],"snakes" [6], geometric splines [7], and finite elements based models [8]. Others are modeled from the statistical perspective. Szeliski [9] introduced the statistical atlas to analyze the shape variation between patients. In order to analyze the shape and appearance variation, principal component analysis (PCA) is widely used $[10,11,12]$. The two most common statistical atlases are the shape atlas [14] and the appearance atlas [13]. The shape atlas uses only geometric information such as landmarks, surfaces (boundaries of 3D objects) or crest lines [3]. The appearance atlas uses both geometric features and intensity of pixels or voxels.

As a key to build atlases, 3D registration has been studied for years in computer vision, but still remains a critical problem in the medical image field due to the geometrical complexity of anatomical shapes, and computational complexity caused by the enormous size of volume data. It has numerous clinical applications such as statistical atlas construction for group study and statistical parameters analysis $[15,16]$, mapping anatomical atlases to individual patient images for disease analysis $[17,18,19]$ and image segmentation $[20,21]$.

Depending on the type of the transformation being involved, registration can be rigid or nonrigid. If the shape has no change between the two images, registration should be rigid, such as in intra-subject inter-modality (same patient; different imaging system) registration where images are captured at the same time. However, when we take into account the time, i.e., when two images are captured at different time, as in intra-subject registrations, most are non-rigid due to the shape variation of the anatomical structures caused by swelling, prostate poking, bone fractures, tumor growth changes, intestinal movements etc. In addition, inter-subject (different patients) registrations are usually non-rigid because of the local anatomical difference between patients. So far non-rigid (also known as deformable) registration is still a challenging problem [22].

Non-rigid registration is used to find a non-rigid transformation from one 3D surface to the reference surface by minimizing the distance between two surfaces. In general, a non-rigid transformation is represented by a global rigid or affine transformation plus a local non-linear deformation, which can be represented by radial basis functions (RBF) [23], octree-spline [24], thin-plate spline [25, 15], geometric splines [7], finite elements [8], or free form B-spline [12], etc. To evaluate the results of registration, different similarity measurements will be selected according to different image features and imaging modalities. For example, sum of squared distance (SSD) is usually used for geometric features [26], but correlation coefficients (CC) [27], Ratio Image Uniformity (RIU) [28], and mutual information (MI) [29] are used for intensity features. Registration can be simplified given known correspondences, for example using markers [30]. Nevertheless, markers are not allowed to be used or even available in many scenarios. Alternate estimation of correspondences and transformations are widely used for both rigid cases [26] and non-rigid cases [15, 25, 31]. Moreover, with the increase of the data size and geometrical complexity, multi-resolution strategy has been adopted to the registration framework [19, 32, 33]. Sparse matrices are also used to handle the computational complexity [34].

To build a femur statistical atlas given partial 3D surfaces, we developed a two-level approach inspired by Chui and Rangarajan's thin-plate spline based algorithm [25] and the previous multi- 
resolution work [33]. Since Chui and Rangarajan's algorithm [25] is not able to handle more than 2000 3D points [34], we broke down registration into a two-level process to deal with both computational and geometrical complexity. We first applied Chui and Rangarajan's algorithm [25] to the simplified low-resolution surfaces. To improve the efficiency, instead of successively matching each resolution from coarse to fine, we directly propagated the correspondences from low resolution to high resolution by interpolation. A local refine procedure was introduced for both low-resolution and high-resolution surfaces to improve matching. Finally we applied PCA to the aligned surfaces to construct the femur atlas. Fig. 1 illustrates the flowchart of our two-level framework.

\section{TWO-LEVEL FRAMEWORK}

\subsection{Mesh Simplification - Decrease Resolution}

Garland's quadric error metrics (QEM) based mesh simplification [35] method was used to compute low-resolution surfaces. QEM is based on iterative contraction of vertex pairs. The cost of contraction is represented by a quadric error and the whole process is an iterative minimization of the quadric error. Since QEM provides a fast, simple way to guide the entire process with relatively minor storage costs, the simplification step is extremely fast.

An important parameter involved in the simplification process is the number of vertices in the lowresolution surfaces. To maintain the tradeoff between the accuracy and efficiency, an appropriate number is selected based on a series of leave-one-out experiments (See details in Sec. 4).

\subsection{Low-Resolution Non-Rigid Registration: Point-to-Point}

We applied Chui and Rangarajan's non-rigid registration method [25] to the simplified surfaces. With this method, fuzzy correspondences and a smoother optimization process can be achieved. A dual update strategy conjuncted with a deterministic annealing technique is adopted to estimate the correspondences and transformation alternately. The non-rigid transformation is parameterized using thin-plate splines to generate a smooth spatial mapping.

Initial Alignment Given the particularity of the femur data, we need to do a pre-alignment to get rid of the effect caused by the missing shape. We have CT-scanned 87 different patients all with healthy femur using the same scanner. Given most surgeries are applied on the joints, only the top portion including the femoral head and the bottom portion including the condyles were scanned to build surfaces.

As Fig. 2 shows, the bottom portion of the femur is used as an example. The surface $Y$ has more femur shaft but less shaft remains on the the surface $X$. If we just align both centers as in previous work [25], experiments show that the registration process will be very slow and may not converge in several cases. The reason is that a part of the surface $Y$ (as bounded by blue in Fig. 2) has no counterpart on the surface $X$. Therefore, in order to improve upon the process, we decided to estimate the pseudo center of $Y$ instead of the true center. And then the pose of two surfaces are 
estimated and aligned.

The height of $X$ is used to estimate the pseudo height of $Y$. Assuming the axis $\mathcal{Z}$ is in the scan direction from the knee to hip, we selected those points bounded by the pseudo height of $Y$ (denoted by black in Fig. 2) to estimate the pseudo center $\kappa_{Y^{\prime}}$ and the covariance matrices for the point set $\left\{\mathbf{p}_{X}\right\}$ and $\left\{\mathbf{p}_{Y^{\prime}}\right\}$ :

$$
\begin{gathered}
\left\{\mathbf{p}_{Y^{\prime}}\right\}: \\
\kappa_{X}=\frac{1}{N_{X}} \sum \mathbf{p}_{X} \\
\kappa_{Y^{\prime}}=\frac{1}{N_{Y^{\prime}}} \sum \mathbf{p}_{Y^{\prime}} \\
\Psi_{X}=\frac{1}{N_{X}-1}\left[\mathbf{p}_{X}^{1}-\kappa_{X}, \cdots, \mathbf{p}_{X}^{N_{X}}-\kappa_{X}\right] \cdot\left[\mathbf{p}_{X}^{1}-\kappa_{X}, \cdots, \mathbf{p}_{X}^{N_{X}}-\kappa_{X}\right]^{\prime} \\
\Psi_{Y^{\prime}}=\frac{1}{N_{Y^{\prime}}-1}\left[\mathbf{p}_{Y^{\prime}}^{1}-\kappa_{Y^{\prime}}, \cdots, \mathbf{p}_{Y^{\prime}}^{N_{Y^{\prime}}}-\kappa_{Y^{\prime}}\right] \cdot\left[\mathbf{p}_{Y^{\prime}}^{1}-\kappa_{Y^{\prime}}, \cdots, \mathbf{p}_{Y^{\prime}}^{N_{Y^{\prime}}}-\kappa_{Y^{\prime}}\right]^{\prime}
\end{gathered}
$$

where $N_{Y^{\prime}}$ is the number of points $\left\{\mathbf{p}_{Y^{\prime}}\right\}$ in $Y$ which satisfy $\left(z_{Y}-\min z_{Y}\right)<\left(\max z_{X}-\min z_{X}\right)$. We can solve for the principle axes by decomposing the covariance matrix using the moment analysis:

$$
\Psi_{X}=\mathbf{U}_{X} \Lambda_{X} \mathbf{U}_{X}{ }^{\prime}, \Psi_{Y^{\prime}}=\mathbf{U}_{Y^{\prime}} \Lambda_{Y^{\prime}} \mathbf{U}_{Y^{\prime}}{ }^{\prime}
$$

Each column of $\mathbf{U}_{X}$ represents a principle axis of the point set $\left\{\mathbf{p}_{X}\right\}$, and $\mathbf{U}_{Y^{\prime}}$ for $\left\{\mathbf{p}_{Y^{\prime}}\right\}$. As Fig. 2 shows, three axes represent the pose of the point set: red for $\left\{\mathbf{p}_{X}\right\}$ and green for $\left\{\mathbf{p}_{Y^{\prime}}\right\}$. The rotation from coordinates of $X$ to $Y^{\prime}$ is computed by $\mathbf{U}_{Y^{\prime}} \cdot \mathbf{U}_{X^{\prime}}$. We thus applied a rigid transformation $\left[\mathbf{U}_{Y^{\prime}} \cdot \mathbf{U}_{X}{ }^{\prime} \mid\left(\kappa_{Y^{\prime}}-\kappa_{X}\right)\right]$ to the point set $\left\{\mathbf{p}_{X}\right\}$ and the two point sets are finally aligned. Experiment shows the rate of convergence has been improved from $78 \%$ to $95.2 \%$ by such alignment.

\subsection{Local Refine Procedure: Point-to-Surface}

The result of registration from last section can be further improved by minimizing the point-tosurface distance. This idea is illustrated by Fig. 3. $\mathbf{x}_{i}$ is a vertex on the deformed surface $X$, whose corresponding vertex on the surface $Y$ is $\mathbf{y}_{i}$. The neighboring triangles which share the same vertex $\mathbf{y}_{i}$ are $S_{1}, S_{2}$ and $S_{3}$. We can compute the distance from $\mathbf{x}_{i}$ to each neighboring triangle (the distance computed from the vertex $\mathbf{x}_{i}$ to the plane where the triangle lies), i.e. $d_{1}, d_{2}$ and $d_{3}$. If any of them is smaller than $d_{0}=\left\|\mathbf{x}_{i}-\mathbf{y}_{i}\right\|$, we will use the corresponding projected point to replace $\mathbf{y}_{i}$ to achieve a smaller surface distance.

For those cases where different vertices on the surface $X$ correspond to the same surface point on $Y$, we will assign this corresponding surface point to the vertex on $X$ with the smallest distance and mark it unavailable to other vertices on $X$.

\subsection{Low-Resolution to High-Resolution Interpolation}

Once we obtained the correspondences in low-resolution $\left(X^{L}\right.$ and $\left.Y^{L}\right)$, we propagated them to high resolution $\left(X^{H}\right.$ and $\left.Y^{H}\right)$ by interpolation. The surface interpolation method is a derivative of methods known jointly as "moving least squares" [36]. Radial basis functions (RBF), finite element, multivariate spline such as thin-plate spline (2D bivariate spline) and triharmonic thin-plate spline, are popular techniques used in surface interpolation. Carr et al. [37] applied multivariate splines method into radial basis functions by using splines as kernel functions. In this work we 
chose Gaussian kernel due to its simple mathematical representation and less restrictions on nodes [37]. More specifically, we used a linear affine function plus a series of radial basis functions (RBFs) to construct the interpolation function:

$$
\mathbf{y}_{i}^{L}=g\left(\mathbf{x}_{i}^{L}\right)=\underbrace{\mathbf{c}_{\mathbf{1}} \cdot\left[\varphi\left(\left\|\mathbf{x}_{i}^{L}, \mathbf{x}_{1}^{L}\right\|\right), \cdots, \varphi\left(\left\|\mathbf{x}_{i}^{L}, \mathbf{x}_{N}^{L}\right\|\right)\right]^{\prime}+\mathbf{c}_{\mathbf{2}}+\mathbf{c}_{\mathbf{3}} \cdot \mathbf{x}_{i}^{L}}_{g\left(\mathbf{x}_{i}^{L}\right)}
$$

where $\mathbf{x}_{i}^{L}$ is a vertex on the low-resolution surface $X^{L}$, whose correspondence on the low-resolution surface $Y^{L}$ is $\mathbf{y}_{i}^{L}, i=1,2, \cdots, N\left(N\right.$ is the number of vertices on $\left.X^{L}\right) . \mathbf{x}_{i}^{L}$ and $\mathbf{y}_{i}^{L}$ are both $3 \times 1$ vectors with three coordinates. $\mathbf{c}_{\mathbf{1}}$ is a $3 \times N$ coefficient matrix of radial basis functions. $\varphi\left(\left\|\mathbf{x}_{i}^{L}, \mathbf{x}_{j}^{L}\right\|\right)$ is a symmetric radial basis function. We selected a Gaussian kernel $\varphi\left(\mathbf{u}_{\mathbf{i}}, \mathbf{u}_{\mathbf{j}}\right)=$ $\exp \left(-\left\|\mathbf{u}_{\mathbf{i}}-\mathbf{u}_{\mathbf{j}}\right\| / 0.5\right)$, as suggested by [38]. $\mathbf{c}_{\mathbf{2}}$ and $\mathbf{c}_{\mathbf{3}}$ are coefficients for the affine transformation. $\mathbf{c}_{2}$ is a $3 \times 1$ vector and $\mathbf{c}_{3}$ is a $3 \times 3$ matrix. Given $N$ correspondences, there are $N$ equations for each axis $(\mathcal{X}, \mathcal{Y}$ and $\mathcal{Z})$ :

$$
\underbrace{\left[\begin{array}{ccccc}
\varphi\left(\mathbf{x}_{1}^{L}, \mathbf{x}_{1}^{L}\right) & \cdots & \varphi\left(\mathbf{x}_{1}^{L}, \mathbf{x}_{N}^{L}\right) & 1 & \mathbf{x}_{1}^{L^{T}} \\
\varphi & & & \\
\varphi\left(\mathbf{x}_{N}^{L}, \mathbf{x}_{1}^{L}\right) & \cdots & \varphi\left(\mathbf{x}_{N}^{L}, \mathbf{x}_{N}^{L}\right) & 1 & \mathbf{x}_{N}^{L^{T}}
\end{array}\right]}_{\mathbf{P}^{k}} \cdot \underbrace{\left[\begin{array}{c}
\mathbf{c}_{\mathbf{1}}^{\mathbf{k}^{\mathbf{T}}} \\
\mathbf{c}_{\mathbf{2}}^{\mathbf{k}^{\mathbf{T}}} \\
\mathbf{c}_{\mathbf{3}}^{\mathbf{k}^{\mathbf{T}}}
\end{array}\right]}_{\mathbf{c}^{k}}=\underbrace{\left[\begin{array}{c}
\mathbf{y}_{1}^{L^{k}} \\
\vdots \\
\mathbf{y}_{N}^{L^{k}}
\end{array}\right]}_{\mathbf{y} \mathbf{v}^{k}}
$$

where $\mathbf{c}_{\mathbf{1}}{ }^{k}, \mathbf{c}_{\mathbf{2}}{ }^{k}$ and $\mathbf{c}_{\mathbf{3}}{ }^{k}$ denote the $k^{\text {th }}$ row of $\mathbf{c}_{\mathbf{1}}, \mathbf{c}_{\mathbf{2}}$ and $\mathbf{c}_{\mathbf{3}}$, respectively. $\mathbf{y}_{i}^{L^{k}}$ denotes the $k^{\text {th }}$ row of $\mathbf{y}_{i}^{L}, k$ can be 1,2 or 3 , corresponding to the axis $\mathcal{X}, \mathcal{Y}$, and $\mathcal{Z}$. There are $3 N$ equations in total:

$$
\mathbf{P}=\left[\mathbf{P}^{1}, \mathbf{P}^{2}, \mathbf{P}^{3}\right]^{\prime}, \mathbf{c}=\left[\mathbf{c}^{1}, \mathbf{c}^{2}, \mathbf{c}^{3}\right]^{\prime}, \mathbf{y}=\left[\mathbf{y}^{1}, \mathbf{y}^{2}, \mathbf{y}^{3}\right]^{\prime}
$$

$\mathbf{P}$ is a $3 N \times(N+4)$ matrix. In order to ensure smooth interpolation, additional orthogonality constraints $\sum_{i} \mathbf{x}_{i}^{L^{T}} \mathbf{c}_{\mathbf{1}, \mathbf{i}}=0$ [39] were added to Eq. 5, where $\mathbf{c}_{\mathbf{1}, \mathbf{i}}$ denotes the $i^{\text {th }}$ column of $\mathbf{c}_{\mathbf{1}}$ :

$$
\underbrace{\left[\begin{array}{ccccc} 
& \mathbf{P} & \\
\mathbf{x}_{1}^{L} & \mathbf{x}_{2}^{L} & \cdots & \mathbf{x}_{N}^{L} & \mathbf{0}_{4 \times 4}
\end{array}\right]}_{\mathbf{Q}} \cdot \mathbf{c}=\underbrace{\left[\begin{array}{c}
\mathbf{y} \\
\mathbf{0}_{4 \times 1}
\end{array}\right]}_{\mathbf{w}}
$$

The least-squares solution for this linear system, $\mathbf{Q} \mathbf{c}=\mathbf{w}$, is given by $\mathbf{c}=\left(\mathbf{Q}^{T} \mathbf{Q}\right)^{-1} \mathbf{Q}^{T} \mathbf{w}$.

Finally, the correspondence of a vertex $\mathbf{x}_{j}^{H}$ in the high-resolution surface $X^{H}$ can be computed by using Eq. 4: $\mathbf{y}_{j}^{H}=g\left(\mathbf{x}_{j}^{H}\right)$, for $j=1, \cdots, M$ ( $M$ is the number of vertices on $\left.X^{H}\right)$. We also refined the resulted high-resolution surface by the procedure described in Sec. 2.3.

\section{FEMUR ATLAS CONSTRUCTION}

Given the aligned surfaces, rigid pose alignments are applied to eliminate the effect of imaging poses [40] prior to atlas construction. Suppose we have $K$ aligned surfaces and each surface is represented by a $3 M \times 1$ vector $\mathbf{v}_{i}(i=1, \cdots, K)$, where $M$ is the number of points on each surface and 3 denotes three coordinates $\mathcal{X}, \mathcal{Y}$, and $\mathcal{Z}$. We computed the mean vector $\kappa$ and covariance matrix $\Psi$, and then applied PCA to the data set: 


$$
\begin{gathered}
\kappa=\frac{1}{K} \sum \mathbf{v}, \Psi=\frac{1}{M-1}\left[\mathbf{v}^{1}-\kappa, \cdots, \mathbf{v}^{K}-\kappa\right] \cdot\left[\mathbf{v}^{1}-\kappa, \cdots, \mathbf{v}^{K}-\kappa\right]^{\prime} \\
\Psi=\mathbf{U} \Lambda \mathbf{U}^{\prime}
\end{gathered}
$$

Therefore, any surface model in the data set can be represented by a mean vector plus a linear combination of principal components (modes):

$$
\mathbf{v}_{i}=\kappa+\mathbf{U} \boldsymbol{\eta}_{\boldsymbol{i}}
$$

where $\boldsymbol{\eta}_{\boldsymbol{i}}$ is a $K \times 1$ coefficient vector obtained by projecting $\mathbf{v}_{i}$ onto each principal axis. New surface models, not included in the data set, can be generated by manipulating the coefficient $\boldsymbol{\eta}_{\boldsymbol{i}}$.

\section{SELECTION OF SIMPLIFICATION PARAMETERS}

In the two-level framework, the resolution of the simplified surfaces affects the final result. The fewer the number of points, the faster low-resolution registration is achieved but results in highresolution registration are less accurate. To maintain both accuracy and efficiency, an appropriate number is selected based on a series of leave-one-out experiments. For some extreme cases with symmetry shapes, the fewer points may result in a perfect match in the low resolution yet a misalignment in the high-resolution. Thereby not only the resolution parameter but also some landmarks are needed to handle such ambiguity. Since the shape of the femur is complex enough, we do not consider such extreme situations. Thus we do not require any landmarks in our method. Let $N_{\text {ref }}^{\text {low }}$ denote the number of vertices in the low-resolution surfaces. For each surface $\mathbf{v}_{i}(i=$ $1, \cdots, K), K=87$, we used other $K-1$ surfaces to construct the atlas using Eq. 8 and 9. Let $\mathbf{U}_{i}^{S}$ denote the first $S$ columns of the principal component matrix $\mathbf{U}_{i}$, which consists of $95 \%$ of the shape variation. Then $\mathbf{v}_{i}(i=1, \cdots, K)$ can be reconstructed by this atlas:

$$
\widetilde{\mathbf{v}}_{i}=\kappa+\mathbf{U}_{i}^{S} \mathbf{U}_{i}^{S^{T}}\left(\mathbf{v}_{i}-\kappa\right)
$$

We compared the surface distance between the original surface $v_{i}$ and the reconstructed surface $\widetilde{v_{i}}$ by computing the surface mean error and root mean square error. We repeated this procedure for each surface and computed the average mean error and RMS error based on $K$ leave-one-out experiments:

$$
\overline{d_{m}^{t}}=\frac{1}{K} \sum_{i} d_{m}\left(v_{i}, \widetilde{v}_{i}\right), \overline{d_{R M S}^{t}}=\frac{1}{K} \sum_{i} d_{R M S}\left(v_{i}, \widetilde{v}_{i}\right)
$$

By tuning the number $N_{r e f}^{\text {low }}$, we compared the error $\overline{d_{m}^{t}}$ and $\overline{d_{m}^{t}}$, and processing time $\overline{T^{t}}$ for the two-level registration as well. Fig. 5 shows when $N_{r e f}^{\text {low }} \geq 0.2 \% N_{r e f}^{\text {high }}, \overline{d_{m}^{t}}$ will be less than $1 \mathrm{~mm}$, which is a practical number for clinical applications. Fig. 6 shows when $N_{r e f}^{\text {low }} \geq 0.3 \% N_{\text {ref }}^{\text {high }}$, the average processing time of the two-level registration will exceed 5 mins $(2.4 \mathrm{GHz}$ Pentium PC with 1GB RAM). So $N_{r e f}^{\text {low }}=0.2 \% N_{r e f}^{\text {high }}$ was finally used in our algorithm to build femur atlases and estimate the error distributions illustrated in Fig. 13. 


\section{EXPERIMENTS AND DISCUSSION}

We have CT-scanned 87 different patients all with healthy femur in both West Pennsylvania Hospital (Pittsburgh, PA) and Shadyside Hospital (Pittsburgh, PA) over a period of years. There are 53 males and 34 females. 43 are left femurs and 44 are right ones. The patients' age ranges from 39 to 78 and their femur length ranges from $400 \mathrm{~mm}$ to $540 \mathrm{~mm}$ (Fig. 4 shows the data distribution in terms of the age and femur length). The CT volumes were segmented to provide triangulated surface models using Marching Cube (MC) algorithm. All surface models were smoothed by the method proposed in [41]. Given most surgeries are applied on the joints, only the top portion including the femoral head and the bottom portion including the condyles were scanned to build surfaces. Thereby each femur data has two separated surfaces containing the femoral head and condyles, respectively.

\subsection{Evaluation of Two-level Registration}

Figs. 7-12 show an example of how the surfaces distance is decreased in each step of the two-level framework. We use the bottom portion of the femur as an example given its important relationship with the knee. In this case we have two high-resolution surface $X^{H}$ (21130 vertices, 42256 triangles, $65.8 \mathrm{~mm}$ in z-axis) and $Y^{H}$ (26652 vertices, 53300 triangles, $105.9 \mathrm{~mm}$ in z-axis) (Patient $\mathrm{X}$ is a 79 years old female, with $472.6 \mathrm{~mm}$ femur length; Patient $\mathrm{Y}$ is a 53 years old female, with 477.6mm femur length). We first computed the point-to-surface distance from $X^{H}$ to $Y^{H}$ [42]:

$$
d\left(p, Y^{H}\right)=\min _{p^{\prime} \in Y^{H}}\left\|p-p^{\prime}\right\|_{2}, p \in X^{H}
$$

where $\|\cdot\|_{2}$ is Euclidean norm. The HSV color (HSV stands for hue, saturation, value) of each vertex on $X^{H}$ denotes the distance $d\left(p, Y^{H}\right)$. We also computed the mean error $d_{m}\left(X^{H}, Y^{H}\right)$ and root mean square (RMS) error $d_{R M S}\left(X^{H}, Y^{H}\right)$ between $X^{H}$ and $Y^{H}$ based on Eq. 13:

$$
\begin{aligned}
d_{m}\left(X^{H}, Y^{H}\right) & =\frac{1}{\left|X^{H}\right|} \sum_{p \in X^{H}} d\left(p, Y^{H}\right) d X^{H} \\
d_{R M S}\left(X^{H}, Y^{H}\right) & =\sqrt{\frac{1}{\left|X^{H}\right|} \sum_{p \in X^{H}} d\left(p, Y^{H}\right)^{2} d X^{H}}
\end{aligned}
$$

1. Fig. 7 shows the high-resolution surfaces $X^{H}$ and $Y^{H}$. With respect to the bounding box diagonal of $Y^{H}(158.5 \mathrm{~mm})$, the mean error is $6.49 \%$ and root mean square error is $7.70 \%$.

2. Fig. 8 shows the low-resolution surfaces $X^{L}$ (169 vertices, 334 triangles) and $Y^{L}(213$ vertices, 422 triangles) after simplification. With respect to the bounding box diagonal of $Y^{L}(158.3 \mathrm{~mm})$, the mean error is $6.53 \%$ and root mean square error is $7.74 \%$.

3. Fig. 9 shows the deformed low-resolution surfaces $X^{L(1)}$ and $Y^{L}$ after applying Chui and Rangarajan's non-rigid registration [25] to $X^{L}$. With respect to the bounding box diagonal of $Y^{L}$, the mean error is $1.68 \%$ and root mean square error is $2.13 \%$. The surface distance has been significantly decreased by Chui and Rangarajan's non-rigid registration method [25].

4. Fig. 10 shows the deformed low-resolution surfaces $X^{L(2)}$ and $Y^{L}$ after applying a local refine process to $X^{L(1)}$. With respect to the bounding box diagonal of $Y^{L}$, the mean error 
is $0.68 \%$ and root mean square error is $1.42 \%$, which shows local point-to-surface refining procedure can decrease surface distance further.

5. Fig. 11 shows the interpolated high-resolution surfaces $X^{H(1)}$ and $Y^{H}$ after applying the interpolation to $X^{H}$. With respect to the bounding box diagonal of $Y^{H}$, the mean error is $1.65 \%$ and root mean square error is $2.10 \%$. The reason why the surface distance slightly increases by interpolation is: only $0.80 \%$ of vertices on $X^{H(1)}$ have correspondences computed from low-resolution registration, other correspondences were obtained by interpolation.

6. Fig. 12 shows the deformed high-resolution surfaces $X^{H(2)}$ and $Y^{H}$ after applying a local refining process to $X^{H(1)}$. With respect to the bounding box diagonal of $Y^{H}$, the mean error is $0.28 \%$ and root mean square error is $1.26 \%$, which once again demonstrates that local point-to-surface refining procedure is helpful for decreasing surface distance.

\subsection{Comparison to Other Registration Methods}

We compared our registration algorithm with the classical iterative closet point (ICP) method since it is widely used in many medical image registration problems. Given 87 training surfaces, the distribution of RMS error and mean error of the two approaches are showed in Fig. 13. For RMS error, they are centered between $0.6-1.0 \mathrm{~mm}$ (the maximum is $1.4 \mathrm{~mm}$ ) in our method, but in ICP the range is between $3.1-6.5 \mathrm{~mm}$ (the maximum is $8.5 \mathrm{~mm}$ ). For mean error, they are centered between $0.5-0.7 \mathrm{~mm}$ (the maximum is $0.9 \mathrm{~mm}$ ) in our method, but in ICP the range is $2.5-6.5 \mathrm{~mm}$ (the maximum is $7.5 \mathrm{~mm})$. It is obvious that the two-level non-rigid registration has a better performance than ICP does.

Since Chui and Rangarajan's thin-plate spine (TPS) based method [25] cannot deal with the complexity of our data, we cannot directly compare two methods. Alternatively, we noticed from Fig. 6 that our method only needs 5 minutes or less to match any size of surfaces with less than 200,000 vertices. However, their method costs 5 minutes for 350 vertices, 10 minutes for 460 vertices, 20 minutes for 610 vertices, etc. It is obvious that our algorithm significantly improves efficiency. To maintain a good accuracy for practical applications, a best simplification parameter was selected based on a series of leave-one-out experiments (see details in Sec. 4).

\subsection{Evaluation of Atlases}

We built three atlases for the femoral head, condyles and the entire femur, respectively. Fig. 14 shows the shape variation in the first three modes of the femoral head atlas. The color shapes (pink and blue) are used to highlight the local variation. E.g. the size of the femoral head (denoted by the pink circle) gradually reduces along the first mode, but remains almost the same along the second mode. The greater trochanter (denoted by the blue area) becomes longer but narrower along the first mode. It remains the same length but becomes wider along the third mode.

Fig. 15 shows the shape variation in the first three modes of the condyles atlas. We noticed that the top of the condyles (denoted by the blue area) changes a lot along each mode. This is because each scan was cut differently. The top area can only be estimated from the reference surface without any 
refining from the original surfaces. The lateral epicondyle (denoted by the blue point on the right) becomes larger along the first mode, compared to the medial epicondyle (denoted by the blue point on the left). It is similar to the third mode but the change is smaller. The condyles become longer but narrower along the second mode.

Figs. 16-17 show the shape variation in the first two modes of the entire femur atlas. For the first mode, the femoral head and greater trochanter follow the similar change as Fig. 14 shows, and the condyles become longer and narrower as Fig. 15 shows. The atlas of the entire femur behaves in a similar way as the individual portion does for the first mode. For the second mode, the greater trochanter becomes shorter but it remains the same as in Fig. 14. The condyles become much longer but they keep the same length as in Fig. 15. It means that the second dominant mode in the individual atlas may not be the second dominant mode in the conjunction case.

Fig. 18 shows the shape variation encoded in modes of each atlas. To keep $95 \%$ of shape variation we only need 26 modes for the femoral head atlas, 49 modes for the condyles atlas and 20 modes for the entire femur atlas. The reason why the condyles atlas needs more modes to keep most of the shape variation is because the training surfaces of the bottom portion of the femur change a lot in shape (i.e. Each surface contains different length of femur shaft).

\section{CONCLUSION}

In this paper we developed a two-level framework to efficiently tackle non-rigid registration challenges caused by geometrical and computational complexity. Our work is mainly inspirited by Chui and Rangarajan's method [25] but performs much faster and requires less memory. Experiments demonstrate that our method significantly improves efficiency of registration without decreasing accuracy of atlases. Shape variation learned from both femur atlases can be used for clinical studies and diagnosis in future. Moreover, femur atlases can be used as a statistical prior for shape reconstruction from other imaging modalities such as endoscopes, and also for the segmentation of 3D surfaces from DICOM images.

\section{References}

[1] McInerney T, Terzopoulos D. Deformable models in medical image analysis: a survey. Medical Image Analysis 1996;1(2):91-108.

[2] Subsol G, Thirion JP, Ayache N. First steps towards automatic building of anatomical atlas. INRIA, France 2216, March 1994.

[3] Subsol G, Thirion JP, Ayache N. A general scheme for automatically building of anatomical atlas: application to a skull atlas. INRIA, France 2586, May 1995.

[4] Broit C. Optimal registration of deformed images. Ph.D. Dissertation, Computer and Information Science Dept., Univ. of Penn., Philadelphia, PA 1981.

[5] Bajcsy R, Kovacic S. Multiresolution elastic matching. Proc. CVGIP 1989;46:1-21. 
[6] Kass M, Witkin A, Terzopoulos D. Snakes: Active contour models. Int. J. Computer Vision 1988;1(4):321-331.

[7] Farin G. Curves and surfaces for CAGD. Academic Press, New York 1993.

[8] Park J, Mataxas D, Young A, Axel L. Deformable models with parameter functions for cardiac motion analysis from tagged MRI data. IEEE Trans. on Medical Imaging 1996;15:278289.

[9] Szeliski R. Bayesian modeling of uncertainty in low-level vision. Int. J. Computer Vision 1990;5:271-301.

[10] Cootes TF, Beeston C, Edwards GJ, Taylor CJ. A unified framework for atlas matching using active appearance models. Proc. Information Processing in Medical Imaging (IPMI) 1999. p 322-333.

[11] Meller S, Kalender WA. Building a statistical shape model of the pevis. International Congress Series 2004;1268:561-566.

[12] Rueckert D, Frangi AF, Schnabel JA. Automatic construction of 3-D statistical deformation models of the brain using nonrigid registration. IEEE Trans. on Medical Imaging 2003;22(8):1014-1025.

[13] Cootes TF, Taylor CJ. Statistical models of appearance for medical image analysis and computer vision. Proc. SPIE Image Processing 2001;(4322):238-248.

[14] Cootes TF, Taylor CJ, Cooper D, Graham J. Active shape models: their training and application. Computer Vision and Image Understanding 1995;61(1):38-59.

[15] Chui H, Rangarajan A, Zhang J, Leonard CM. Unsupervised Learning of an atlas from unlabeled point-sets. IEEE Trans. on PAMI 2004;2:160-172.

[16] Hill DLG, Batchelor PG, Holden M, Hawkes DJ. Medical image registration. Physics in Medicine and Biology 2001:46(3):1-45.

[17] Chen M. 3-D Deformable registration using a statistical atlas with applications in medicine. Ph.D. Dissertation, Robotics Institute, School of Computer Science, Carnegie Mellon University, Pittsburgh, PA 1999.

[18] Fleute M, Lavallée S, Desbat L. Integrated Approach for Matching Statistical Shape Models with Intra-operative 2D and 3D Data. Proc. MICCAI 2002;2:364-372.

[19] Jaume S, Macq B, Warfield SK. Labeling the brain surface using a deformable multiresolution mesh. Proc. MICCAI 2002. p 451-458.

[20] Rohlfing T, Russakoff DB, Maurer CR. Performance-based classifier combination in atlasbased image segmentation using expectation-maximization parameter estimation. IEEE Trans. on Medical Imaging 2004;23(8):983-994. 
[21] Straka M, Cruz AL, Dimitrov LI, Sramek M, Fleischmann D, Groller E. Bone segmentation in CT-angiography data using a probabilistic atlas. Proc. Vision, Modeling and Visualization (VMV) 2003. p 505-512.

[22] Dawant BM. Non-rigid registration of medical images: purpose and methods, a short survey. Proc. IEEE Intl. Symp. Biomed. Imag. 2002. p 465-468.

[23] Yu H. Automatic rigid and deformable medical image registration. Ph.D. Dissertation, Mechanical Engineering Department, Worcester Polytechnic Institute, Worcester, MA 2005.

[24] Szeliski R, Lavallee S. Matching 3-D anatomical surfaces with non-rigid deformations using octree-splines. Int. J. Computer Vision 1996;18:171-186.

[25] Chui H, Rangarajan A. A new point matching algorithm for non-rigid registration. Computer Vision and Image Understanding 2003;89(2-3):114-141.

[26] Besl PJ, McKay ND. A method for registration of 3-D shapes. IEEE Trans. on PAMI 1992;14(2):239-256.

[27] Kim J, Fessler JA. Intensity-based image registration using robust correlation coefficients. IEEE Trans. on PAMI 2004;23(11):1430-1444.

[28] Woods RP, Mazziotta JC, Cherry SR. MRI-PET registration with automated algorithm. J. Computer Assisted Tomography 1994;17:536-546.

[29] Wells WM, Viola P, Atsumi H, Nakajima S, Kikinis R. Multi-modal volume registration by maximization of mutual information. Medical Image Analysis 1996;1(1):35-51.

[30] Maurer CR, Fitzpatrick JM, Wang MY, Galloway RL, Maciunas RJ, Allen GG. Registration of head volume images using implatable fiducial markers. IEEE Trans. on PAMI 1997;16:447-462.

[31] Glaunes J, Trouvé A, Younes L. Diffeomorphic matching of distributions: a new approach for unlabelled point-sets and sub-manifolds matching. Proc. Computer Vision and Pattern Recognition (CVPR) 2004;2:712-718.

[32] Ellingsen LM, Prince JL. Deformable registration of CT pelvis images using Mjolnir. Proc. SPIE Medical Imaging Conference 2006;6144:329-337.

[33] Shen D, Davatzikos C. HAMMER: hierarchical attribute matching mechanism for elastic registration. IEEE Trans. on Medical Imaging 2002;21(11):1421-1439.

[34] Papademetris X, Jackowski AP, Schultz RT, Staib LH, Duncan JS. Non-rigid brain registration using extended robust point matching for composite multisubject fMRI analysis. Proc. MICCAI 2003;2879:788-795.

[35] Garland M, Heckbert PS. Surface simplification using quadric error metrics. Proc. SIGGRAPH 1997. p 209-216. 
[36] Yngve G, Turk G. Robust creation of implicit surfaces from polygonal meshes. IEEE Trans. on Vizualization and Computer Graphics 2002;8(4):346-359.

[37] Carr JC, Fright WR, Beatson RK. Surface interpolation with radial basis functions for medical imaging. IEEE Trans. on Medical Imaging 1997;16(1):96-107.

[38] Pighin F, Hecker J, Lischnski D, Szeliski R, Salesin DH. Synthesizing realistic facial expressions from photographs. Proc. SIGGRAPH 1998. p 75-84.

[39] Carr JC, Beatson RK, Cherrie JB, Mitchell TJ, Fright WR, Mccallum BC, Evans TR. Reconstruction and representation of 3D objects with radial basis functions. Proc. SIGGRAPH 2001. p 67-76.

[40] Goryn D, Hein S. On the estimation of rigid body rotation from noisy data. IEEE Trans. on PAMI 1995;17(2):1219-1220.

[41] Desbrun M, Meyer M, Schroder P, Barr AH. Implicit fairing of irregular meshes using diffusion and curvature flow. Proc. SIGGRAPH 1999. p 317-324.

[42] Aspert N, Santa-Cruz D, Ebrahimi T. MESH: measuring errors between surfaces using the hausdorff distance. Proc. IEEE Int. Conf. in Multimedia and Expo (ICME) 2002;1:705-708. 


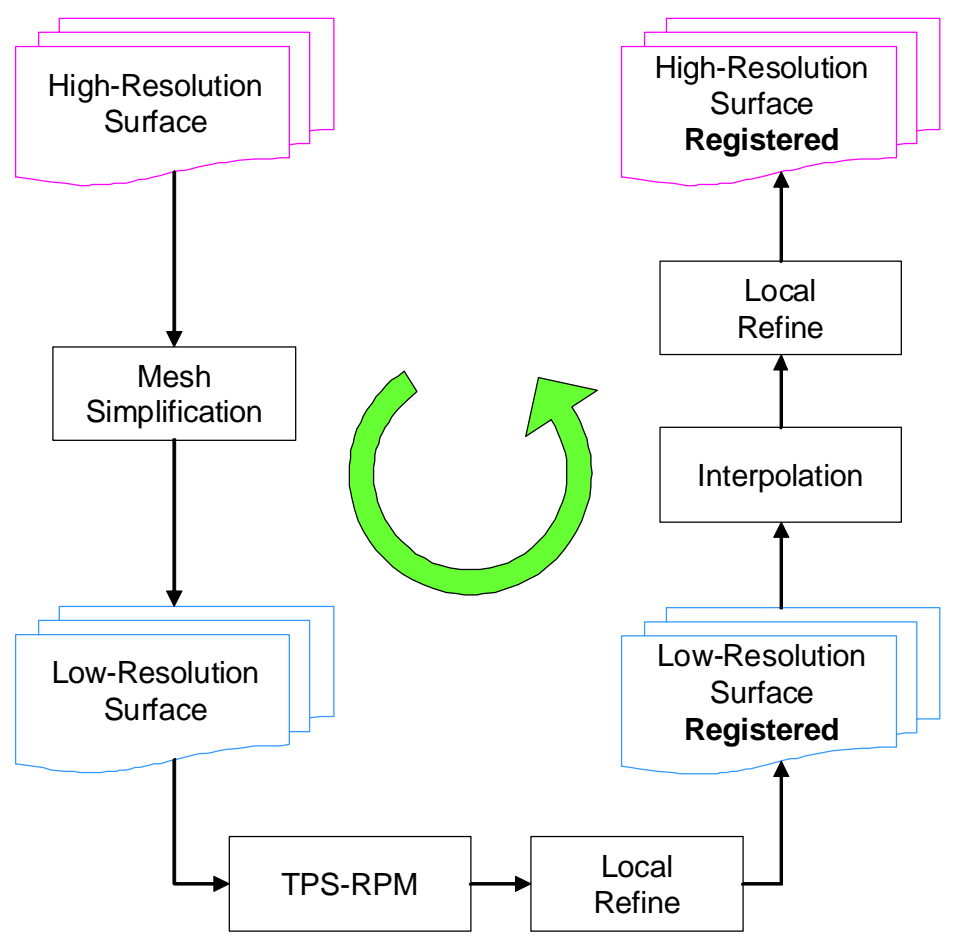

Figure 1: Two-level non-rigid registration framework.

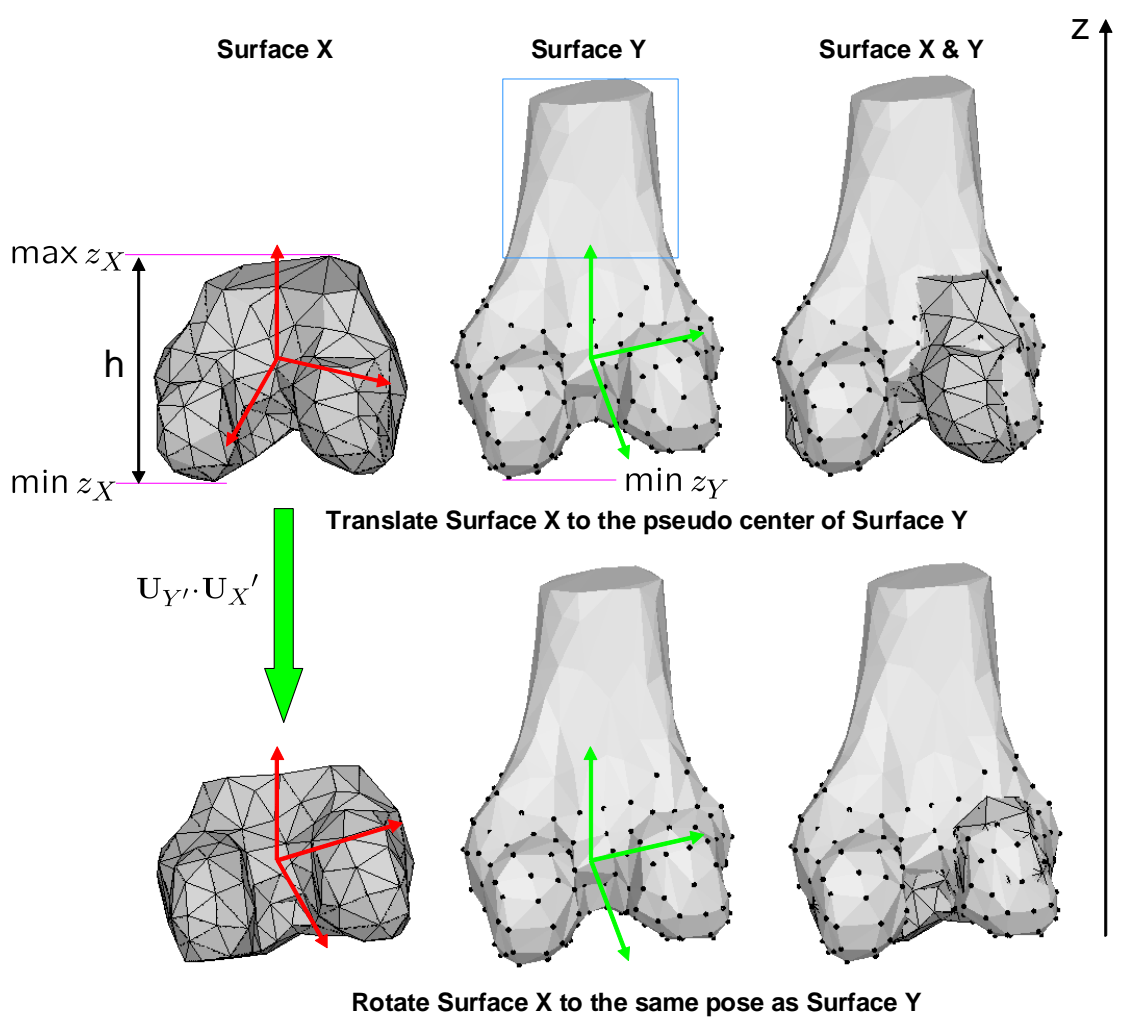

Figure 2: Illustration of the rigid transformation from the surface $\mathrm{X}$ to the surface $\mathrm{Y}$. The first row compares the translated $\mathrm{X}$ with $\mathrm{Y}$. Black points on $\mathrm{Y}$ are used to compute the pseudo center. The second row compares the translated and rotated $\mathrm{X}$ with $\mathrm{Y}$. Red axes represent the pose of $\mathrm{X}$, green axes for $\mathrm{Y}$. 


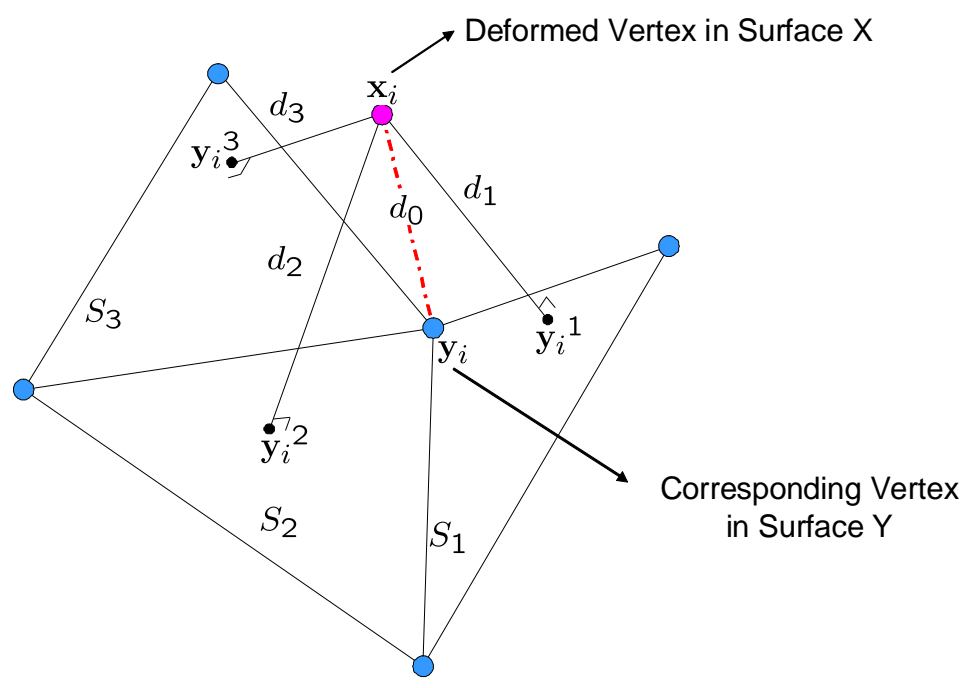

Figure 3: Illustration of the local refine procedure between the vertex and surface. $\mathbf{x}_{i}$ is a vertex on the deformed surface $X$, whose correspondence on the surface $Y$ is $\mathbf{y}_{i}$. The projection of $\mathbf{x}_{i}$ to each triangle $S_{t}$ sharing $\mathbf{y}_{i}$ is denoted by $\mathbf{y}_{i}{ }^{t} . d_{t}$ is the distance between $\mathbf{x}_{i}$ and $\mathbf{y}_{i}{ }^{t} .(t=1,2,3, \cdots)$
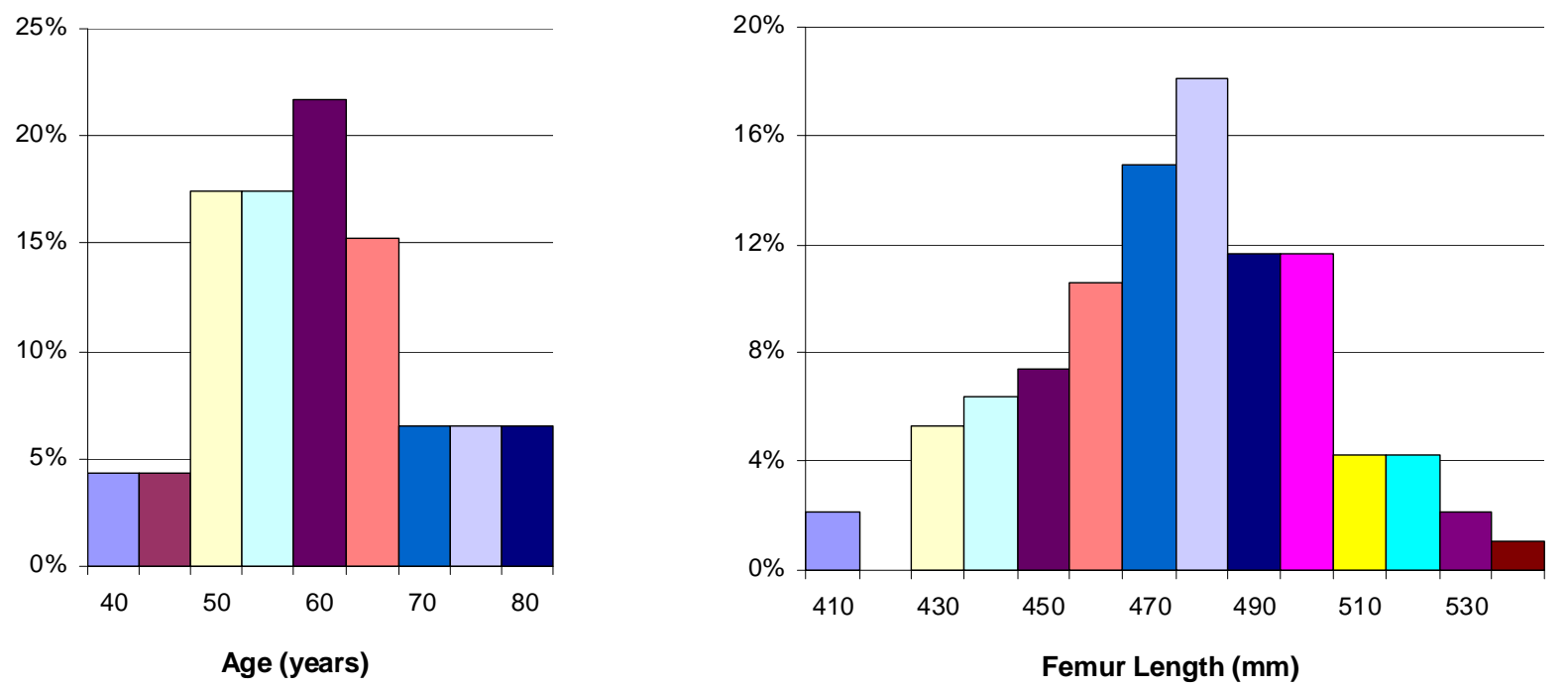

Figure 4: Data distributions in terms of age (left) and femur length (right). 


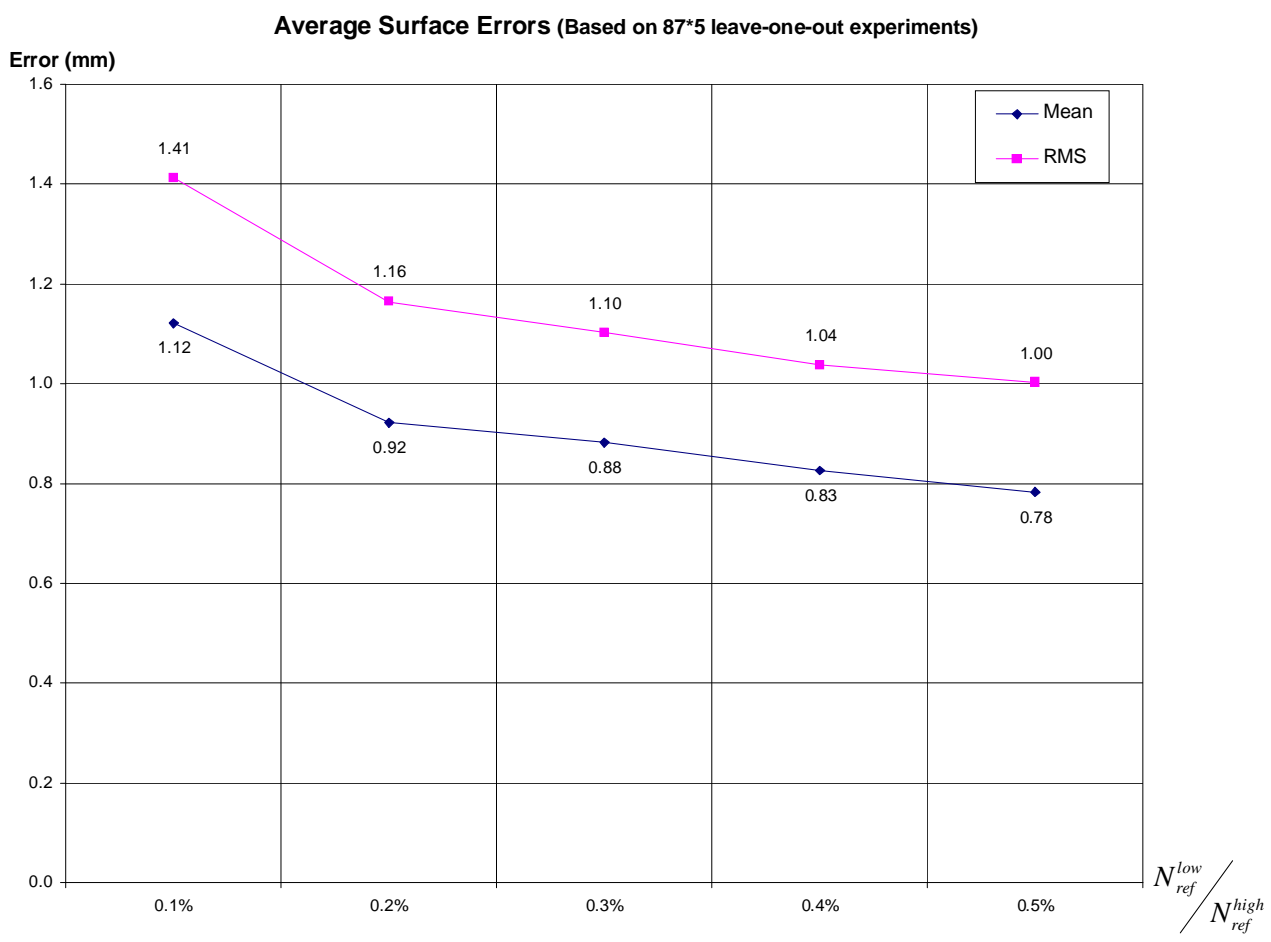

Figure 5: Illustration of the reconstruction errors $\overline{d_{m}}$ and $\overline{d_{R M S}}$ in terms of $N_{r e f}^{\text {low }} / N_{r e f}^{\text {high }}$.

Average Processing Time (Based on $86^{\star} 5$ registration results)

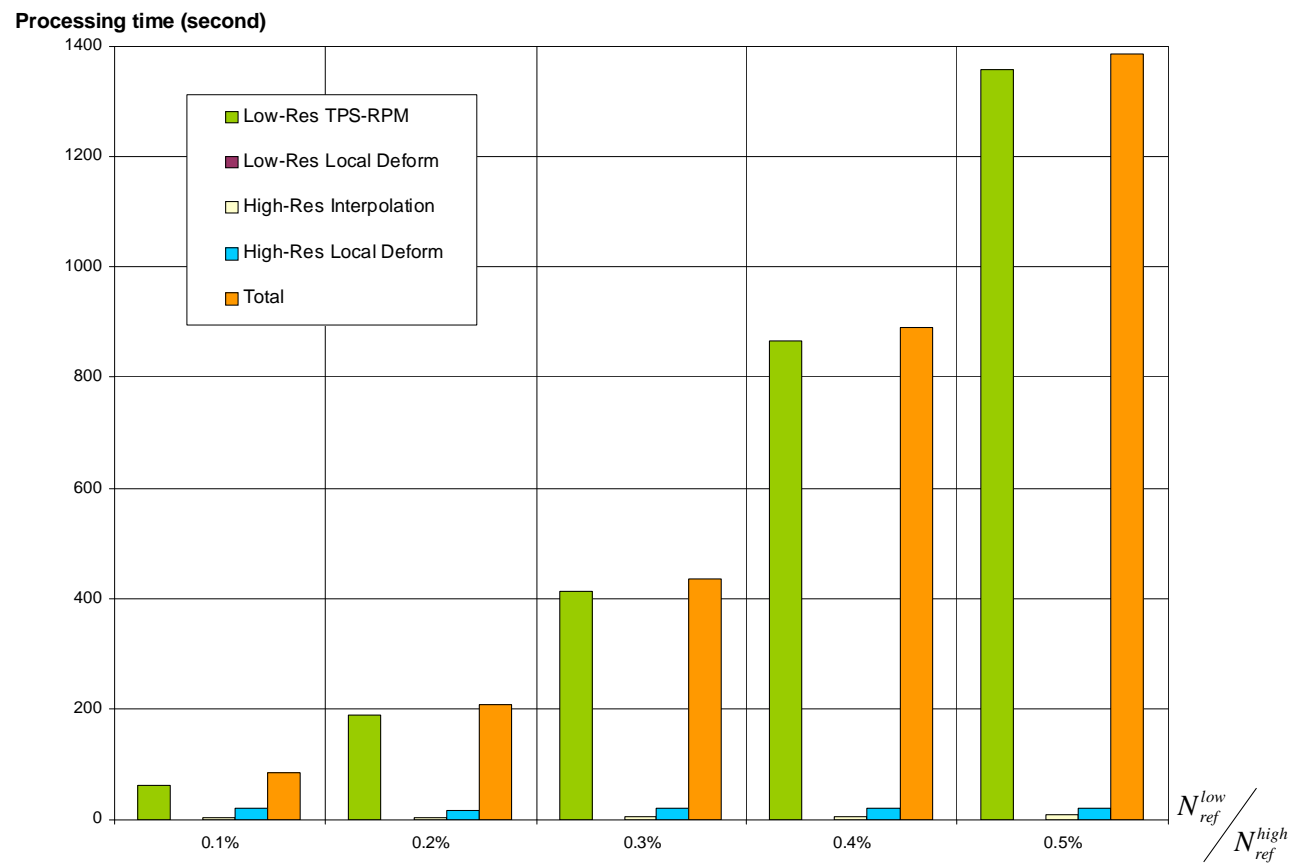

Figure 6: Illustration of the processing time $\bar{T}$ in terms of $N_{r e f}^{\text {low }} / N_{\text {ref }}^{\text {high }}$. 


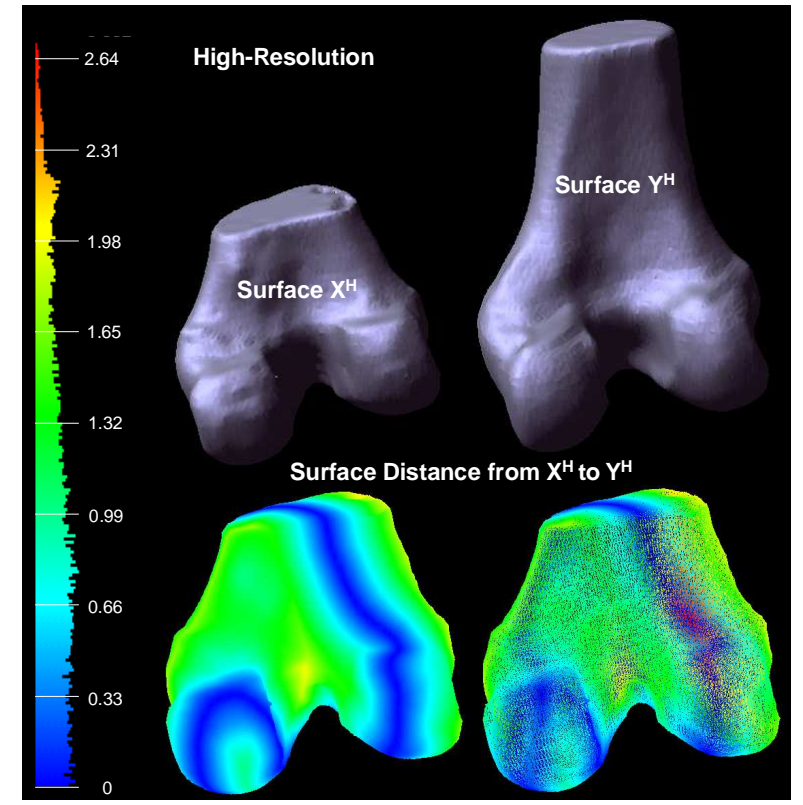

Figure 7: Illustration of the high-resolution surfaces $X^{H}$ and $Y^{H}$. Point-to-surface distances from $X^{H}$ to $Y^{H}$ are illustrated by a HSV color map: the color of each vertex on $X^{H}$ represents the distance $d\left(p, Y^{H}\right), p \in X^{H}$. (Both surface (left) and mesh (right) are showed in the bottom row.)

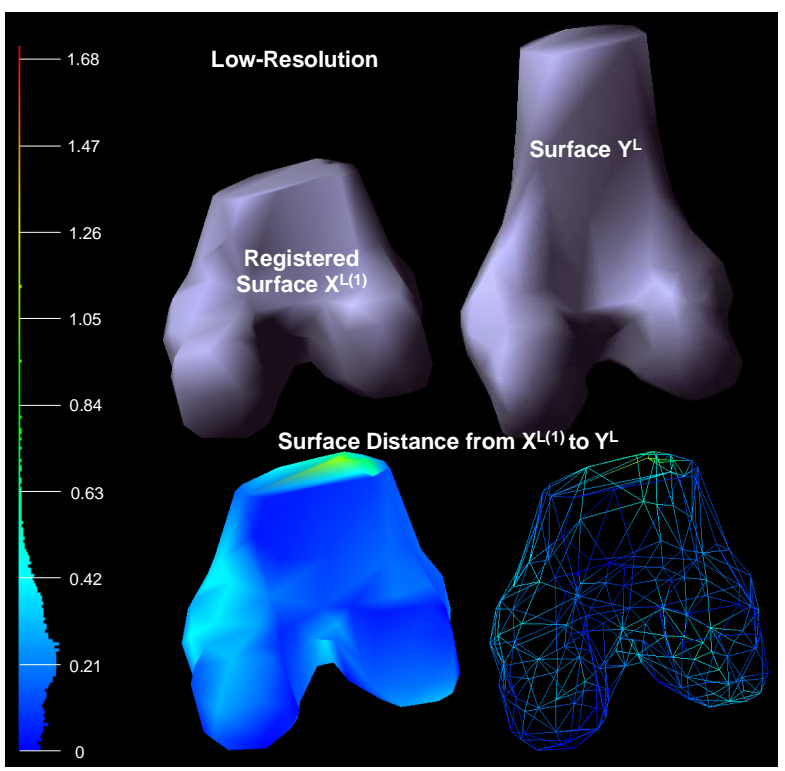

Figure 9: Illustration of the deformed low-resolution surfaces $X^{L(1)}$ and $Y^{L}$ after applying Chui and Rangarajan's non-rigid registration method (see Sec. 2.2) to $X^{L}$. Point-to-surface distances from $X^{L(1)}$ to $Y^{L}$ are illustrated by a HSV color map: the color of each vertex on $X^{L(1)}$ represents the distance $d\left(p, Y^{L}\right), p \in$ $X^{L(1)}$. (Both surface (left) and mesh (right) display modes are showed in the bottom row.)

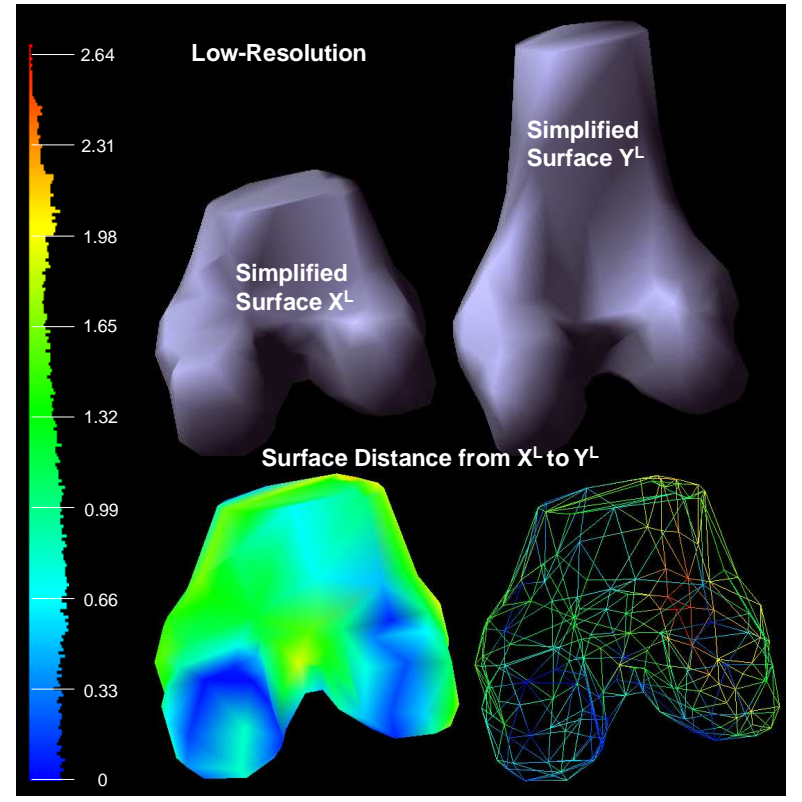

Figure 8: Illustration of the low-resolution surfaces $X^{L}$ and $Y^{L}$ after applying the mesh simplification (see Sec. 2.1) to both $X^{H}$ and $Y^{H}$. Point-to-surface distances from $X^{L}$ to $Y^{L}$ are illustrated by a HSV color map: the color of each vertex on $X^{L}$ represents the distance $d\left(p, Y^{L}\right), p \in X^{L}$. (Both surface (left) and mesh (right) display modes are showed in the bottom row.)

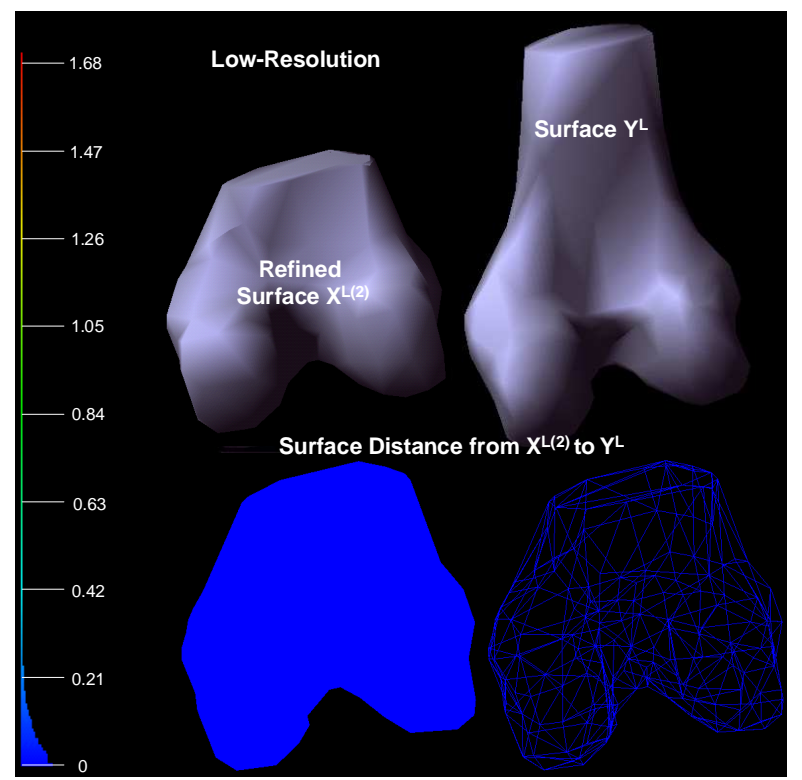

Figure 10: Illustration of the deformed lowresolution surfaces $X^{L(2)}$ and $Y^{L}$ after applying the local refinement ( see Sec. 2.3) to $X^{L(1)}$. Point-tosurface distances from $X^{L(2)}$ to $Y^{L}$ are illustrated by a HSV color map: the color of each vertex on $16 X^{L(2)}$ represents the distance $d\left(p, Y^{L}\right), p \in X^{L(2)}$. (Both surface (left) and mesh (right) display modes are showed in the bottom row.) 


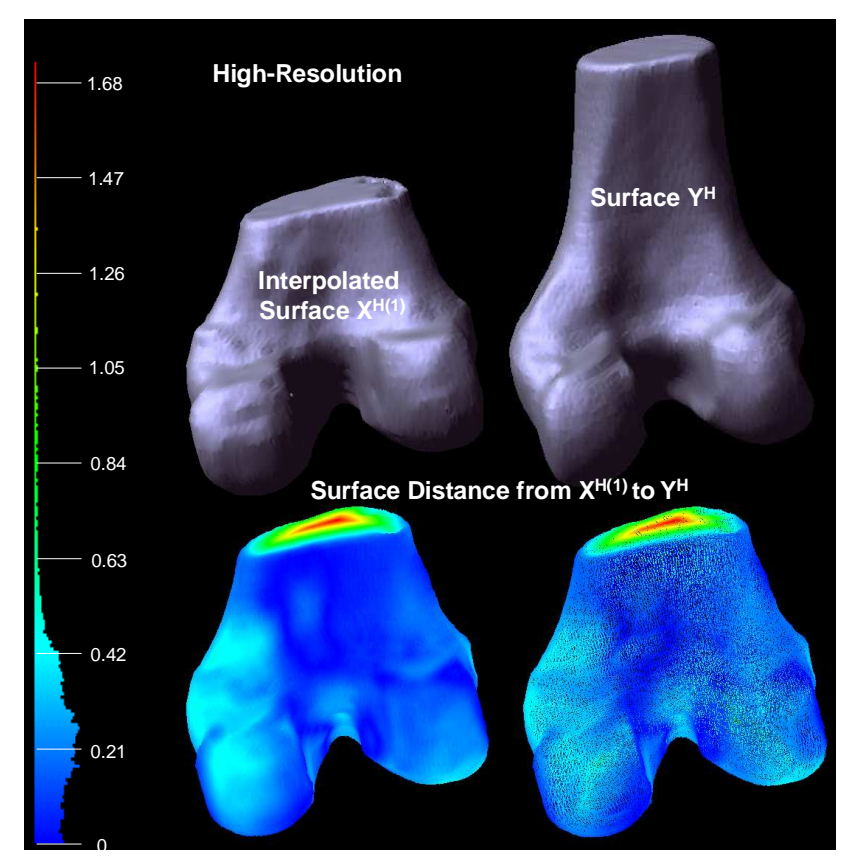

Figure 11: Illustration of the deformed highresolution surfaces $X^{H(1)}$ and $Y^{H}$ after applying the interpolation (see Sec. 2.4) to $X^{H}$. Point-to-surface distances from $X^{H(1)}$ to $Y^{H}$ is illustrated by a HSV color map: the color of each vertex on $X^{H(1)}$ represents the distance $d\left(p, Y^{H}\right), p \in X^{H(1)}$. (Both surface (left) and mesh (right) display modes are showed in the bottom row.)

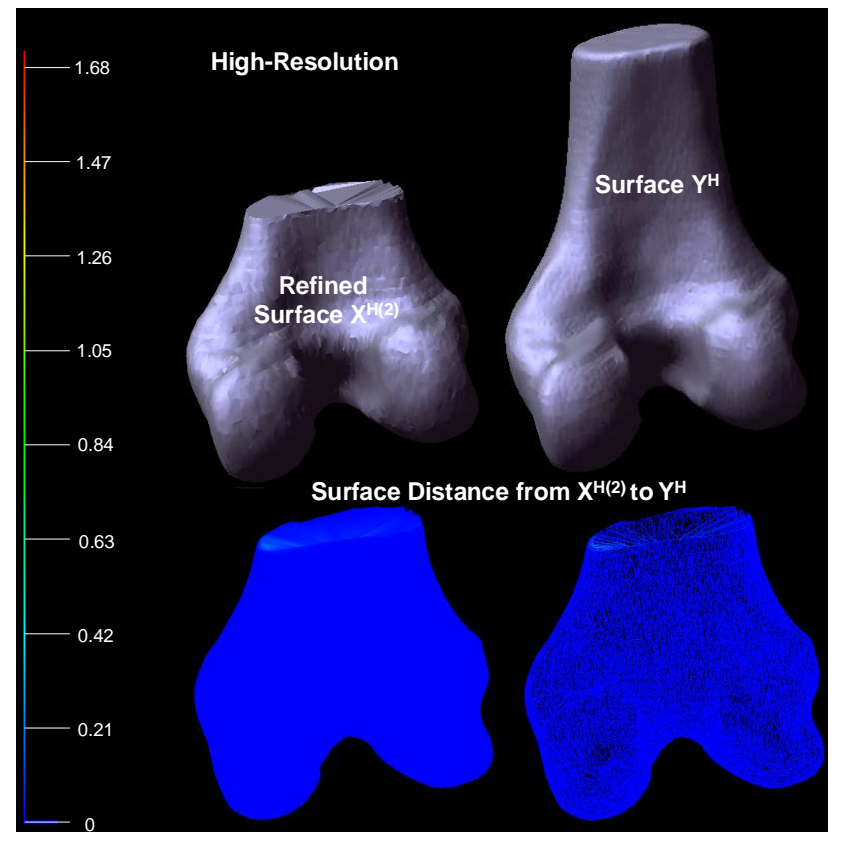

Figure 12: Illustration of the deformed highresolution surfaces $X^{H(2)}$ and $Y^{H}$ after applying the local refinement (see Sec. 2.3) to $X^{H(1)}$. Pointto-surface distances from $X^{H(2)}$ to $Y^{H}$ is illustrated by a HSV color map: the color of each vertex on $X^{H(2)}$ represents the distance $d\left(p, Y^{H}\right), p \in X^{H(2)}$. (Both surface (left) and mesh (right) display modes are showed in the bottom row.) 
RMS Error Distribution for Our Method (Based on 87 patients)
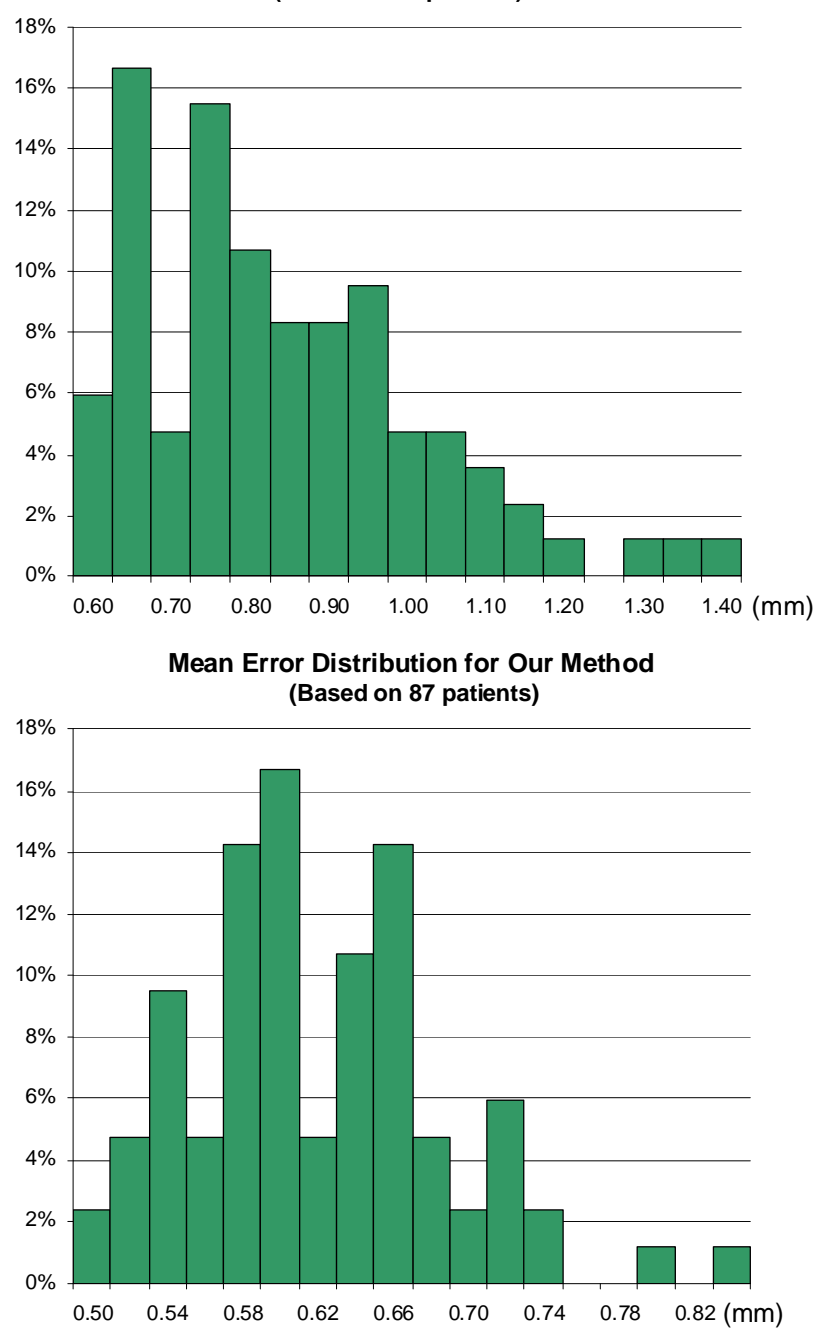

RMS Error Distribution for ICP (Based on 87 patients)

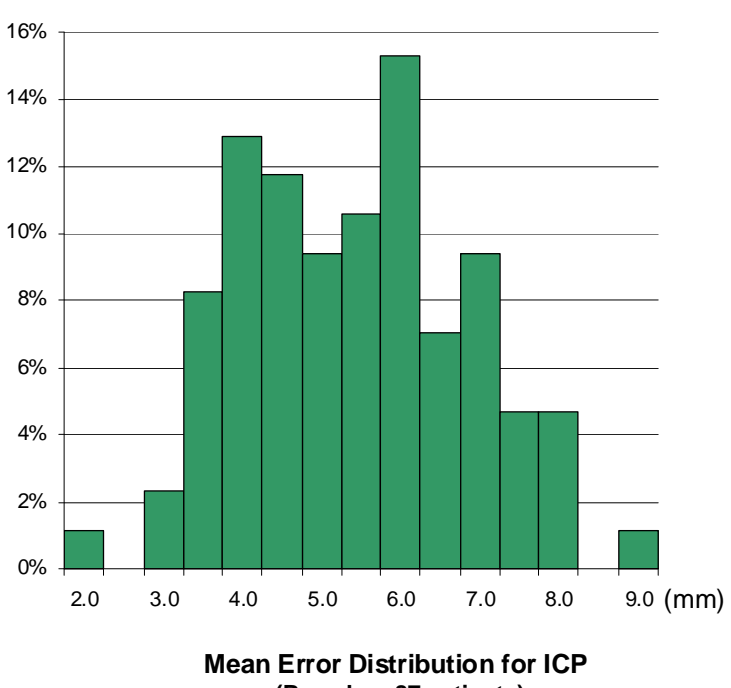
(Based on 87 patients)

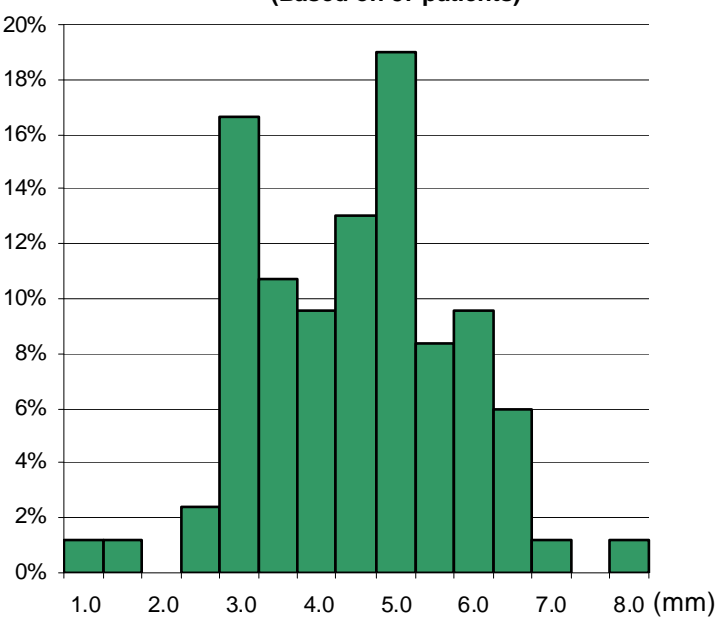

Figure 13: RMS and Mean error distributions from our method and ICP. 

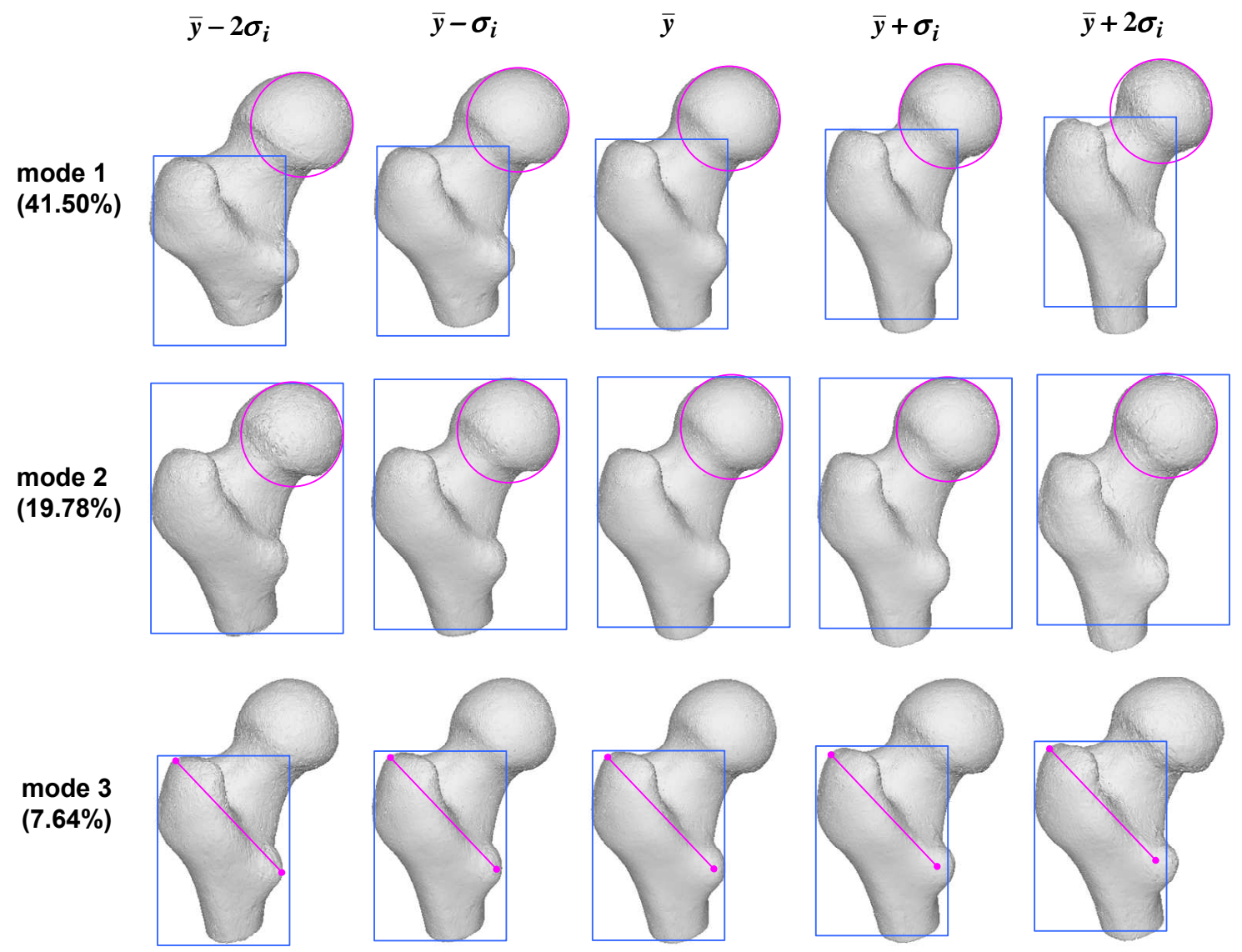

Figure 14: Femoral head atlas: illustration of the shape variation in the first, second and third mode. The first mode encodes $41.50 \%$ of the shape variation, the second and third modes encode $19.78 \%$ and $7.64 \%$ of the shape variation. The color shapes highlight the local shape variation.

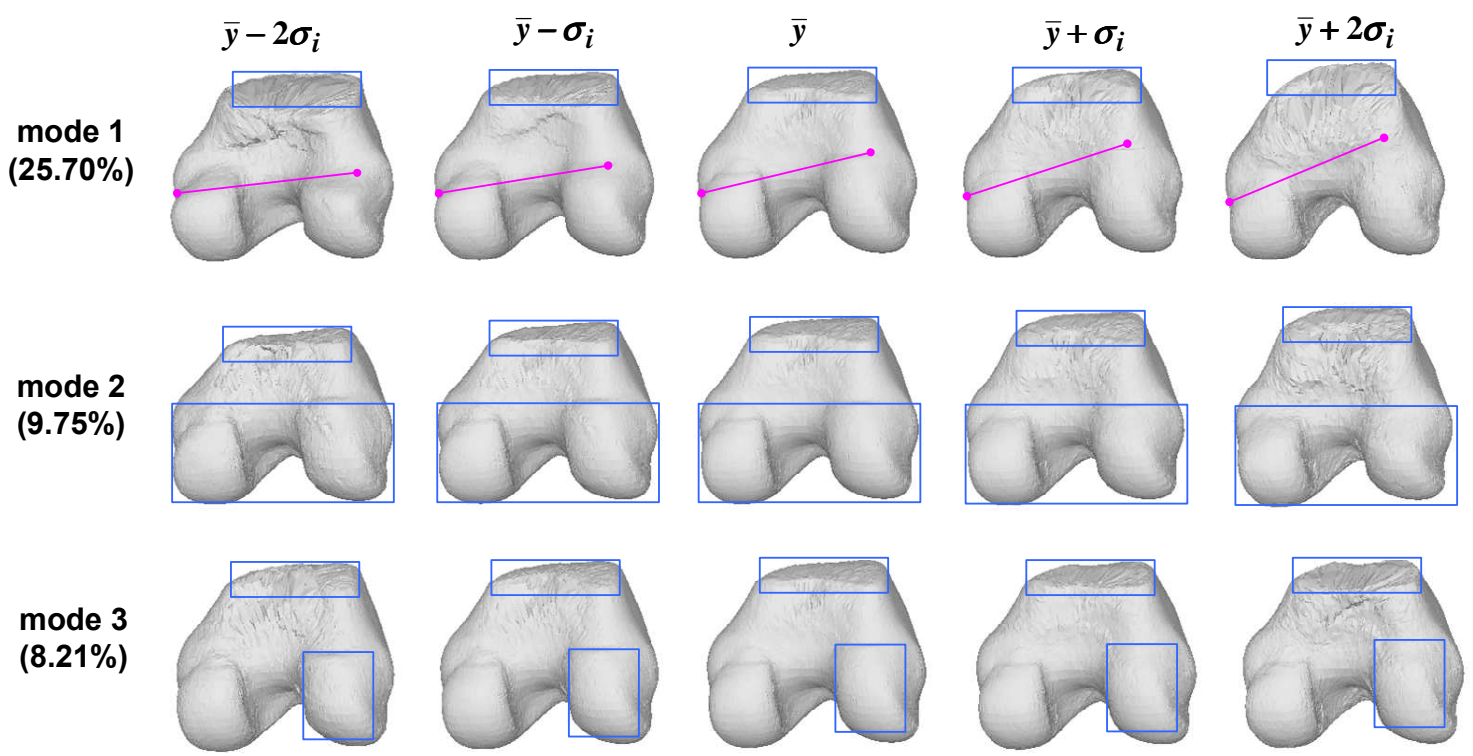

Figure 15: Condyles atlas: illustration of the shape variation in the first, second and third mode. The first mode encodes $25.70 \%$ of the shape variation, the second and third modes encode $9.75 \%$ and $8.21 \%$ of the shape variation. The color shapes highlight the local shape variation. 

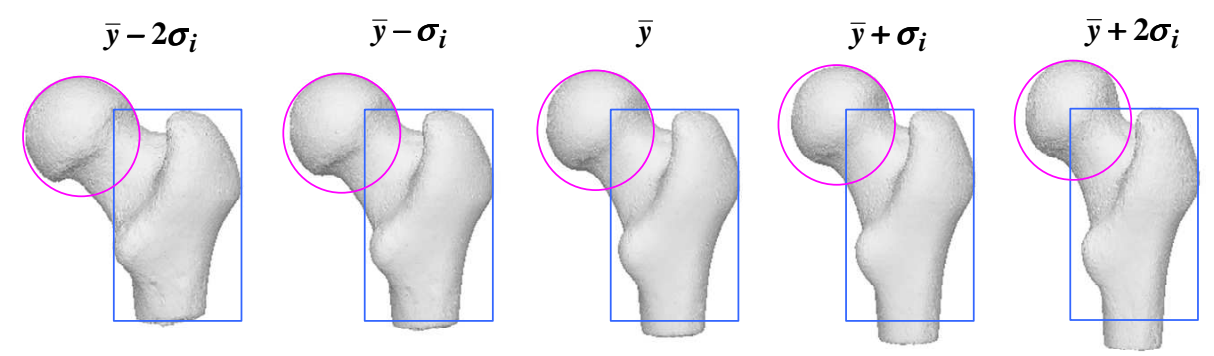

mode 1

(27.67\%)
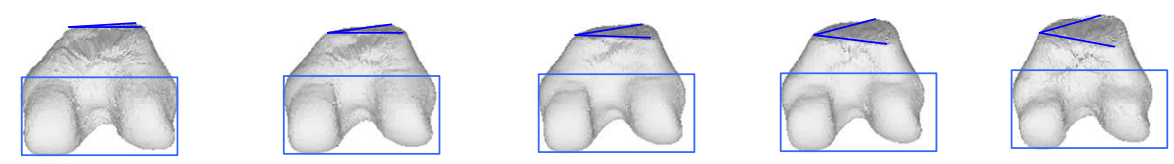

Figure 16: Femur atlas: $27.67 \%$ of the shape variation is encoded in the first mode. The color shapes highlight the local shape variation.
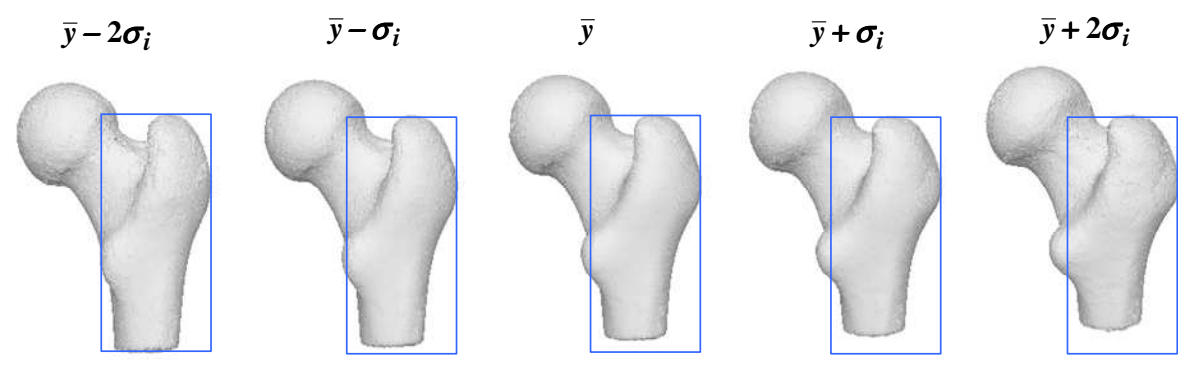

mode 2

$(18.63 \%)$
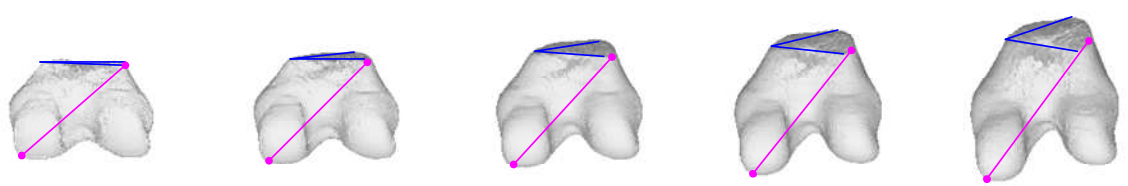

Figure 17: Femur atlas: $18.63 \%$ of the shape variation is encoded in the second mode. The color shapes highlight the local shape variation. 


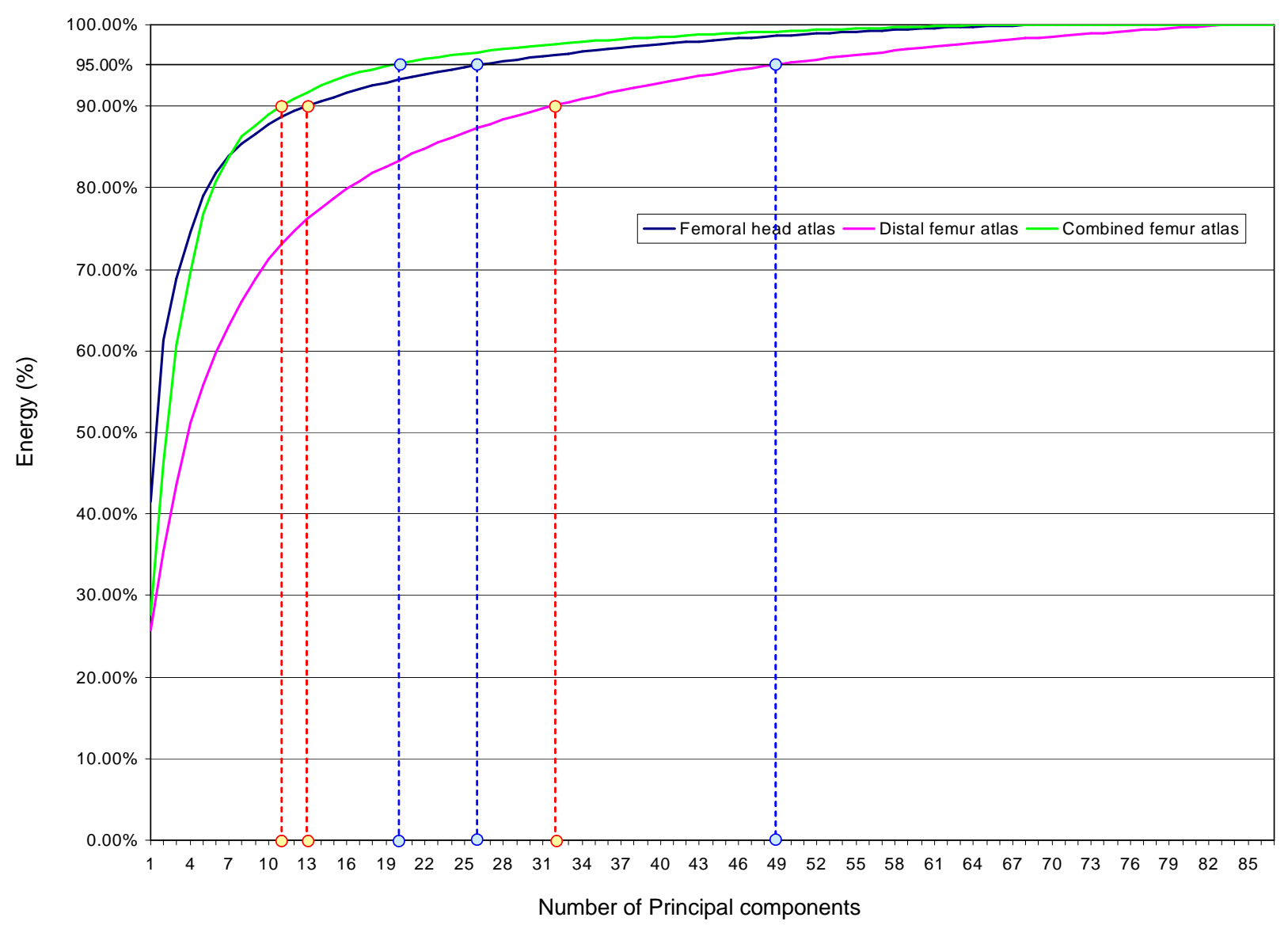

Figure 18: Illustration of the shape variation encoded in modes of the femoral head atlas(blue), condyles atlas(pink) and entire femur atlas(green). 